\title{
Comparison of Short-Term Clinical Outcome in In-Hospital Patients of ST Elevation versus Non ST Elevation Myocardial Infarction
}

\author{
Aparna Rahman ${ }^{1}$, M.M. Zahurul Alam Khan², Md Zahid Alam³ , Shabnam Jahan Hoque ${ }^{4}$,Tunaggina Afrin Khan ${ }^{5}$, \\ Mohammad Shakhawat Hossain6
}

\begin{abstract}
Objective: To compare short term clinical outcome in hospital patient of ST Elevation versus Non ST Elevation Myocardial Infarction.
\end{abstract}

Methodology: This cross sectional observational study was carried out enrolling 100 subjects with ST elevation and Non ST elevation Myocardial Infarction, in the Department of Cardiology, BIRDEM General Hospital, Shahbagh, Dhaka, over a period of six months from January 2012 to June 2012.

Results: Mean age and gender difference was significant between STEMI and non-STEMI. Most common short term clinical outcome was heart failure $(80.95 \%$ vs $75.68 \%)$. Atrial fibrillation was observed in $(4.76 \%$ vs $3.44 \%)$, VT( $2.38 \%$ vs $1.72 \%)$, cardiogenic shock $(31.03 \%$ vs $17.24 \%)$, hypotension $(76.19 \%$ vs $58.62 \%)$, reinfarction $(2.38 \%$ vs $00 \%)$ and death $(14.28 \%$ vs $5.17 \%)$ were observed among ST and Non ST elevation MI respectively. Statistical analysis revealed that all the parameters of short term outcome had significant difference except atrial fibrillation and VT.

Conclusion: It could be concluded that short term outcome were relatively worse in ST elevated MI and to be managed with all possible therapeutic modules.

Key words : STEMI, NSTEMI, Myocardial infarction
Introduction:

Acute coronary syndrome (ACS) refers to a constellation of clinical symptoms caused by acute myocardial ischemia. ${ }^{1}$ Owing to their higher risk for cardiac death or ischemic complications, patients with ACS must be identified among the patients with non-traumatic chest symptoms presenting for emergency evaluation. 2,3 In practice, the terms suspected or possible ACS are often used by medical personnel early in the process of evaluation to describe patients for whom the symptom complex is consistent with ACS but the diagnosis has not yet been conclusively established.4, 5 Patients with ACS are subdivided into two major categories; Unstable angina and acute myocardial infarction. New ST-segment elevation / new onset LBBB on the ECG isdiagnostic of

\footnotetext{
1. Senior Medical Officer, BIRDEM, Dhaka, Bangladesh.

2. Prof. of Cardiology BIRDEM, Dhaka, Bangladesh.

3. Asso. Prof. of Cardiology BIRDEM, Dhaka, Bangladesh.

4. Junior Consultant of Cardiology BIRDEM, Dhaka, Bangladesh.

5. Specialist of Cardiology,United Hospital, Dhaka, Bangladesh.

6. Senior Medical Officer, SMO BIRDEM, Dhaka, Bangladesh. Address of Correspondence: Dr. Aparna Rahman, Senior Medical Officer, BIRDEM, Shahbag, Dhaka, Bangladesh. E-mail: aparnadr28@gmail.com
}

acute ST-elevation myocardial infarction (STE-MI), and ST segment depression, T-wave changes or no ECG abnormalities are cases of non-ST elevation MI. The term STE- ACS encompasses only STEMI. ${ }^{6-9}$

Unstable angina and NSTEMI are considered to be closely related conditions, sharing a common pathogenesis and clinical presentation but differing in severity. ${ }^{1}$ Specifically, NSTEMI is distinguished from unstable angina by ischemia sufficiently severe in intensity and duration to cause irreversible myocardial damage (myocyte necrosis), recognized by the elevation of biomarkers of myocardial injury. ${ }^{10}$ The majority of patients with ST-segment elevation ultimately develop a Q-wave AMI (QMI), whereas a minority develops a non Qwave AMI ( Non-QMI). ${ }^{11}$

Most patients with NSTEMI do not reveal a $Q$ wave in the 12 lead electrocardiogram (ECG) and are subsequently referred to as having sustained Non-QMI; only a minority of NSTEMI patients develop a $Q$ wave and are later diagnosed as QMI. It is important to recognize that ACS is a complex syndrome with a heterogeneous etiology. ${ }^{5}$ 
The prognosis of patients with STEMI has improved considerably over the last decade. The introduction of new therapeutic modalities, including invasive cardiac procedures and new medications, probably play a major role in the favorable outcome of this patients. ${ }^{12,13}$

In different studies on the prognosis of STEMI versus non-STEMI have shown different results. Some studies have shown that patients with non-STEMI have a relatively better in-hospital course and a lower early mortality rate. ${ }^{10}$ Also, patients with non-STEMI have a relatively high prevalence of spontaneous infarct artery reperfusion, smaller infarct size, and relatively low in-hospital mortality, but a higher rate of post-infarction recurrent ischemic events.

\section{Methodology:}

This prospective observational study was done in the Department of Cardiology, BIRDEM General Hospital, Shahbagh, Dhaka during the period of January 2012 to June 2012 with the general objective to compare short term clinical outcome in hospital patient with ST Elevation versus non ST Elevation Myocardial Infarction. During the study period 100 consecutive subjects aged 25-75 years suffering from STEMI and non-STEMI who presented with chest discomfort, palpitation or shortness of breath with either ECG change( ST elevation / depression ,T wave changes) or raised Troponin I were enrolled.Patient with chronic stable angina, unstable angina, non - cardiac chest pain, congenital or valvular cases and shortness of breath other than ischemic heart disease were excluded from our study. Study subjects were collected from admitted patient in CCU referred from emergency department and also from in-patient department of the respective disciplinewith acute coronary syndrome.

The objective of the study was discussed in details with the patients or their attendants before their decision to enroll themselves into the study. Clinical examination, laboratory tests, X-ray, ECG and Echocardiography were done and data collected. Demographic information was prospectively recorded including the subject's age, gender, medical and clinical history, clinical examination and follow up of clinical conditions during hospital stay were assessed and study was conducted. Data were analyzed by using SPSS version 13. Categorical data were expressed as frequency and percentage and continuous data were expressed as mean $\pm S D$. Comparison of mean between two groups were done by Students $t$ test. The level of significance was set at 0.5

\section{Result:}

Total 100 case of STEMI and non-STEMI were evaluated after hospital admission of which 42 with STEMI and 58 with NSTEMI. The male female ratio 1:2 (STEMI) and $1: 1.3$ (NSTEMI). The mean age of STEMI and non-STEMI groups were $48.36 \pm 10.18$ and $51.29 \pm 11.55$ years respectively. Majority of $(16 \% \& 19 \%)$ the respondents (STEMI vs Non-STEMI) were found in the age group of 50-59. Mean age difference was significant between STEMI and non-STEMI. (Table - I)

Clinical findings of the study subjects $(n=100)$ are shown in Table II

Tachycardia was observed in $57.14 \%$ STEMI and $62.06 \%$ non STEMI subjects. Bradycardia was seen in $19.04 \%$ STEMI and $10.34 \%$ non STEMI subjects. Hypotension was higher among nonSTEMI (58.62\%) than STEMI (17.19\%). About $38.09 \%$ subjects with STEMI and 58.62\% subjects with non STEMI had edema. JVP was raised among non STEMI (44.82\%) than STEMI(38.09\%). Bilateral basal crepitation was observed in most $(38.09 \%, 34.48 \%$ in STEMI and non STEMI) of the subjects. Except edema, there was significant difference in different signs between STEMI and non STEMI.

ECG findings of the study subjects $(n=100))$ are shown in Table-III

ST elevation was seen in all STEMI subjects. ST depression was observed in $60.36 \%$ subjects with NSTEMI. Arrhythmia (STEMI vs non STEMI $14.28 \%$, $5.17 \%$ ) was also evident in ECG.

Echocardiographic findings of the study subjects $(n=100)$ are shown in Table-IV

Most common Echocardiographic findings of the subjects were regional wall motion abnormalities (ST vs non ST, $100 \%, 68.96 \%$ ). Majority (ST vs non ST, 9.52\%\%, 27.58\%) of the subjects had d" $40-49 \%$ LV dysfunction. Only $19.04 \%$ in STEMI and $24.23 \%$ in non STEMI had e"60\% LV ejection fraction. Significant difference in findings was observed between two groups except normal LV function.

Short term clinical outcome of the study subjects $(n=100)$ are shown in Table-V.

Most common short term clinical outcome was heart failure (ST vs non ST, $80.95 \%$ vs $75.68 \%$ ). Atrial fibrillation was observed in (ST vs non ST, $4.76 \%$ vs $3.44 \%$ ), VT (ST vs non ST, $2.38 \%$ vs $1.72 \%$ ), cardiogenic shock (ST vs non ST, $31.03 \%$ vs $17.24 \%$ ), hypotension (ST vs non ST, $76.19 \%$ vs $58.62 \%$ ), reinfarction (ST vs non ST, $2.38 \%$ vs $00 \%$ ) and death (ST vs non ST, $14.28 \%$ vs $5.17 \%$ ) were observed among the study subjects. Statistical analysis revealed that all the parameters of short term outcome had significant difference except atrial fibrillation and VT. 
Table-I

Age distribution of the study $(n=100)$

\begin{tabular}{lccc}
\hline Age group (Years) & STEMI $(\mathrm{n}=42) \mathrm{n}(\%)$ & Non-STEMI $(\mathrm{n}=58)$ & p valuen $(\%)$ \\
\hline $25-39$ & $06(6)$ & $11(11)$ & $14(14)$ \\
$40-49$ & $08(8)$ & $19(19)$ & $14(14)$ \\
$50-59$ & $16(16)$ & $51.29 \pm 11.55$ & 0.024 \\
60 and above & $12(12)$ & & \\
Mean \pm SD & $48.36 \pm 10.18$ & & \\
Age range & $33-68$ & & \\
\hline
\end{tabular}

Table-II

Clinical findings of the study subjects $(n=100)$

\begin{tabular}{|c|c|c|c|}
\hline Signs & $\begin{array}{c}\text { STEMI }(n=42) \\
n(\%)\end{array}$ & $\begin{array}{c}\text { Non-STEMI }(\mathrm{n}=58) \\
\mathrm{n}(\%)\end{array}$ & $p$ value \\
\hline \multicolumn{4}{|l|}{ Pulse } \\
\hline Tachycardia & $24(57.14)$ & $36(62.06)$ & 0.001 \\
\hline Bradycardia & $08(19.04)$ & $06(10.34)$ & 0.001 \\
\hline Irregular & $06(14.28)$ & $03(05.17)$ & 0.023 \\
\hline \multicolumn{4}{|l|}{ Blood pressure } \\
\hline Hypertension & $04(09.52)$ & 07 (12.06) & 0.034 \\
\hline Hypotension (SBP< $90 \mathrm{~mm}$ of $\mathrm{Hg}$ ) & $32(17.19)$ & $34(58.62)$ & 0.047 \\
\hline Presence of edema & $20(47.61)$ & $34(58.62)$ & 0.056 \\
\hline Presence of raised JVP & $16(38.09)$ & $26(44.82)$ & 0.045 \\
\hline \multicolumn{4}{|l|}{ Crepitation in lung } \\
\hline Basal zone & $10(23.80)$ & $16(27.58)$ & 0.031 \\
\hline Basal and mid zones & $16(38.09)$ & $20(34.48)$ & 0.037 \\
\hline Whole lung & $06(14.28)$ & $04(06.89)$ & 0.022 \\
\hline
\end{tabular}

Table-III

ECG findings of the study subjects $(n=100)$

\begin{tabular}{lccc}
\hline ECG findings & STEMI $(\mathrm{n}=42) \mathrm{n}(\%)$ & Non-STEMI $(\mathrm{n}=58) \mathrm{n}(\%)$ & $\mathrm{p}$ value \\
\hline ST elevation & $42(100)$ & 00 & 0.001 \\
ST depression & 00 & $35(60.36)$ & 0.001 \\
Within normal limit & 00 & $20(34.48)$ & 0.001 \\
Arrythmia & $06(14.28)$ & $03(05.17)$ & 0.011 \\
\hline
\end{tabular}

Table-IV

Echocardiographic findings of the study subjects $(n=100)$

\begin{tabular}{|c|c|c|c|c|}
\hline \multicolumn{2}{|l|}{$\mathrm{ECHO}$ findings(Common) } & \multirow{2}{*}{$\begin{array}{c}\text { STEMI }(\mathrm{n}=42) \\
\mathrm{n}(\%) \\
40(68.96)\end{array}$} & \multirow{2}{*}{$\begin{array}{c}\text { Non-STEMI }(\mathrm{n}=58) \\
\mathrm{n}(\%) \\
0.001\end{array}$} & \multirow[t]{2}{*}{$p$ value } \\
\hline Regional wall motion abnormality & $42(100)$ & & & \\
\hline & Normal $\geq 60 \%$ & $08(19.04)$ & $14(24.13)$ & 0.648 \\
\hline \multirow[t]{4}{*}{ LV dysfunction } & Fair $\leq 50-59 \%$ & $08(19.04)$ & $10(17.28)$ & 0.001 \\
\hline & Mild $\leq 40-49 \%$ & $04(09.52)$ & $16(27.58)$ & 0.029 \\
\hline & Moderate $\leq 30-39 \%$ & $10(23.80)$ & $08(13.79)$ & 0.011 \\
\hline & Severe $\leq 30 \%$ & $12(28.57)$ & $10(17.28)$ & 0.032 \\
\hline
\end{tabular}


Table-V

In hospital short term clinical outcome of the study subjects

\begin{tabular}{lccc}
\hline Short term clinical outcome & STEMI & Non-STEMI & p-value \\
\hline Heart failure & $34(80.95)$ & $44(75.86)$ & 0.011 \\
Atrial fibrillation & $02(4.76)$ & $02(3.44)$ & 0.351 \\
SVT & $01(2.38)$ & 00 & 0.001 \\
VT & $01(2.38)$ & $01(1.72)$ & 0.424 \\
VF & $02(4.76)$ & 00 & 0.028 \\
Cardiogenic-shock(Defined as persistently low & $18(31.03)$ & $10(17.24)$ & 0.001 \\
SBP<90 mm of Hg with features & & & \\
of tissue hypoperfusion) & $06(14.28)$ & $02(3.44)$ & 0.034 \\
Post-infarct angina & $32(76.19)$ & $34(58.62)$ & 0.012 \\
Hypotension (Defined as SBP<90 mm of Hg) & $01(2.38)$ & 00 & 0.033 \\
Reinfarction & $06(14.28)$ & $03(5.17)$ & 0.027 \\
Death & &
\end{tabular}

\section{Discussion:}

Myocardial infarction comprises a group of symptoms attributed to obstruction of the coronary arteries. The most common symptom prompting diagnosis of myocardial infarction is chest pain, often radiating of the left arm or angle of the jaw, pressure-like in character, and associated with nausea and sweating. Myocardial infarction usually consists of ST elevation myocardial infarction and non ST elevation myocardial infarction. ${ }^{1}$ These types are named according to the appearance of the electrocardiogram (ECG/EKG) as nonST segment elevation myocardial infarction (NSTEMI) and ST segment elevation myocardial infarction (STEMI). ${ }^{2}$ Both ST elevation myocardial infarction and non ST elevation myocardial infarction causes significant mortality and morbidity in acute phase as well as in chronic course of disease. With the aim to compare short term clinical outcome in hospital patient with ST Elevated versus Non ST elevated Myocardial Infarction, this present study was carried enrolling 100 subjects in the Department of Cardiology, BIRDEM General Hospital, Dhaka. The findings of the study are discussed on basis of related previous study concerning the chief objective of the study.

It was observed that mean age of STEMI and non-STEMI groups were $48.36 \pm 10.18$ and $51.29 \pm 11.55$ years respectively with a age range from 33 to 68 years. Majority of $(16 \%, 19 \%)$ the respondents (STEMI vs Non-STEMI) were found in the age group of 50-59. STEMI vs NonSTEMI subjects were found in $12 \%$ and $14 \%$ cases respectively above 60 years age group. Mean age difference was significant between STEMI and nonSTEMI.Burazeri et al (2007) $)^{14}$ found that mean age of the study subjects with STEMI was $59.1 \pm 8.7$ years in their study. In STEMI group male female ratio (1:2). In non-
STEMI group male female ratio (1:1.3). Chi-square test revealed significant difference in gender between two groups. Female predominance in our study may be due to consecutive selection of study subjects and small sample size .

Tachycardia was observed in $57.14 \%$ STEMI and $62.06 \%$ non STEMI subjects. Bradycardia was seen in $19.04 \%$ STEMI and $10.34 \%$ non STEMI subjects. Woo et al ${ }^{15}$ reported that cardinal sign of decreased blood flow to the heart was chest pain experienced as tightness around the chest. This was associated with shortness of breath. Some reported palpitations, anxiety or a sense of impending doom and a feeling of being acutely ill. Other studies also revealed the similar comparable sign and symptoms. ${ }^{16,17}$ Previous studies ${ }^{5,8,10}$ revealed potential complications included pulmonary edema and myocardial reinfarction. Our present study revealed Hypotension was higher among non STEMI (58.62\%) than STEMI (17.19\%). About $38.09 \%$ subjects with STEMI and $58.62 \%$ subjects with non STEMI had edema. JVP was raised among non STEMI (44.82\%) than STEMI (38.09\%). Bilateral basal crepitation was observed in most $(38.09 \%, 34.48 \%$ in STEMI and non STEMI) of the subjects. Except oedema, there was significant difference in different signs between STEMI and non STEMI.

ST elevation was seen in all STEMI subjects. ST depression was observed in $60.36 \%$ subjects with NSTEMI. Arrythmia (STEMI vs non STEMI 14.28\%, 5.17\%) was also evident in ECG. Most common Echocardiographic findings of the subjects were regional wall motion abnormalities (ST vs non ST, 100\%, 68.96\%). Majority (ST vs non ST, $9.52 \% \%, 27.58 \%$ ) of the subjects had d" $40-49 \%$ LV dysfunction. Only $19.04 \%$ in STEMI and $24.23 \%$ in non STEMI had e" $60 \%$ LV dysfunction. Significant difference in findings was observed between two groups except normal LV function. Cannon et al (2002) ${ }^{7}$ 
observed the same findings in subjects with ACS. The incidence rates of STEMI were $21 \%$, whereas the incidence rates of NSTEMI was 32\% (McManus et al $2011)^{18}$ according to ECG. Most common Echocardiographicfindings of the subjects were regional wall motion abnormalities (100\%). Majority (34\%) of the subjects had d" $41-50 \%$ LV dysfunction. Only $11 \%$ had e" $56 \%$ LV dysfunction.

\section{Conclusion:}

From the study result it could be concluded that short term complications were relative grave in ST elevated MI. So, subjects with MI who had ST elevation should be paid extra attention during early management. However further study with a comparative prospective design is required to solve these questions.

\section{References:}

1. Braunwald E, Antman EM, Beasley JW, et al. ACC/ AHA 2002 guideline update for the management of patients with unstable angina and non-ST-segment elevation myocardial infarction- summary article: a report of the American College of Cardiology/ American Heart Association task force on practice guidelines (Committee on the Management of Patients with Unstable Angina). J Am Coll Cardiol. 2002; 40:1366-74.

2. Bertrand $M E$, Simoons $M L$, Fox $K A$, et al. Management of acute coronary syndromes: acute coronary syndromes without persistent ST segment elevation; recommendations of the Task Force of the European Society of Cardiology. Eur Heart J. $2000 ; 21: 1406-32$.

3. Storrow AB, Gibler WB. Chest pain centers: Diagnosis of acute coronary syndromes. Ann Emerg Med. 2000; 35:449-61.

4. The Joint European Society of Cardiology/American College of Cardiology Committee for the redefinition of myocardial infarction. Myocardial infarction redefined-a consensus document of The Joint European Society of Cardiology/American College of Cardiology Committee for the redefinition of myocardial infarction. J Am Coll Cardiol. 2000; 36:959-69.

5. Braunwald E. Unstable angina. A classification. Circulation. 1989;80:410-3.

6. Maseri A, Rebuzzi AG, Cianflone D. Need for a composite risk stratification of patients with unstable coronary syndromes tailored to clinical practice. Circulation. 1997; 96:4141-2.

7. Cannon CP. Evidence-based risk stratification to target therapies in acute coronary syndromes. Circulation. 2002; 106:1588-91.
8. Phibbs B, Marcus F, Marriott HJ, Moss A, Spodick $\mathrm{DH}$. Q-wave versus non-Q wave myocardial infarction: a meaningless distinction. J Am Coll Cardiol 1999; 33:576-82.

9. Haim M, Behar S, Boyko V, Hod H, Gottlieb S. The prognosis of a first $\mathrm{Q}$-wave versus non-Q-wave myocardial infarction in the reperfusion era. Am J Med 2000; 108:381-6.

10. Théroux P, Fuster V. Acute coronary syndromes: unstable angina and non-ST elevation myocardial infarction. Circulation 1998; 97:1195-1206.

11. Frank V, Aguirre FV, Younis LT, Chaitman BR. Early, and 1- year clinical outcome of patients evolving non-ST elevation versus ST elevation myocardial infarction after thrombolysis. Results from the TIMI II study. Circulation 1995;91:2541-2548.

12. Charytan DM, Kuntz RE, Chhabra A, Cutlip DE. Relationship of chronic kidney disease to cardiovascular death and myocardial infarction following coronary stenting. J Nephrol 2006;19: 764-70.

13. Lekakis J, Katsoyanni K, Tricopoulos D. Q-versus non-ST elevation myocardial infarction: clinical characteristics and 6-month prognosis. Clin Cardiol 1984; 7:283-8.

14. Burazeri G, Goda A,Sulo G, Stefa J, Roshi E, Kark JD. Conventional Risk Factors and Acute Coronary Syndrome during a Period of Socioeconomic Transition: Population-based Case-control Study in Tirana, Albania. Croat Med J. 2007; 48(2): 225-33.

15. Woo KM, Schneider Jl. High-risk chief complaints I: chest pain-the big three. Emerg. Med. Clin. North Am. 2009; 27 (4): 685-712,

16. Heidenreich PA, Alloggiamento T, Melsop K, McDonald KM, Go AS, Hlatky MA. The prognostic value of troponin in patients with non-ST elevation acute coronary syndromes: a meta-analysis. J Am Coll Cardiol. 2001; 38(2):478-85.

17. O'Connor RE, Bossaert L, Arntz HR, Brooks SC, Diercks D, Feitosa-Filho G, et al. Part 9: acute coronary syndromes: 2010 International Consensus on Cardiopulmonary Resuscitation and Emergency Cardiovascular Care Science With Treatment Recommendations. Circulation. 2010; 122:422-65.

18. McManus DD, Gore J, Yarzebski J, Spencer F, Lessard D, Goldberg RJ. Recent trends in the incidence, treatment, and outcomes of patients with STEMI and NSTEMI. Am J Med. 2011; 124(1): 40-7. 